\title{
Application of propensity scores to explore the effect of public reporting of medicine use information on rational drug use in China: a quasi-experimental design
}

Xiaopeng Zhang, Lijun Wang and Xinping Zhang*

\begin{abstract}
Background: Transparency has become a hottest topic and a growing movement in the health care system worldwide. This study used a quasi-experimental design method to explore whether public reporting of medicine use information can improve rational drug use.

Methods: 20 township hospitals and 274 doctors of City Y in Hubei Province, China were divided into the intervention and control groups on the basis of their characteristics. In the intervention group, the values and rankings of the average expenditure per prescription, percentage of prescriptions requiring antibiotics and percentage of prescriptions requiring injections of each hospital and doctor were publicly released to patients and doctors in an appropriate format monthly. Data were gathered both four months before and after the intervention. Propensity score matching (PSM) was used to minimize the observed covariate (gender, age, experience, education level, title, and monthly income) differences in the doctors' characteristics. 108 pairs of doctors were obtained after PSM. Chi-square test and t-test were employed to explore the effect of public reporting of medicine use information on rational drug use. The study was approved by the Committee of Tongji Medical College, Hua Zhong University of Science and Technology (IORG No: IORG0003571).
\end{abstract}

Results: In baseline, the average expenditure per prescription of the 274 doctors was 42.82 RMB yuan (USD 6.97), the percentage of prescriptions requiring antibiotics was $63.00 \%$, and the percentage of prescriptions requiring injections was $70.79 \%$, all higher than the average of Hubei Province and the standard recommended by WHO. Before the intervention all the three indicators were all comparable $(p>0.05)$, whereas after the intervention, a significant difference $(p<0.05)$ was found for the percentage of prescriptions requiring injections between the intervention (64.66\%) and control groups (70.52\%).

Conclusions: Irrational drug use remains a policy issue in township hospitals in the study area. We demonstrated that publicly reporting medicine use information could decrease the percentage of prescriptions requiring injections in township hospitals in China, but this effect was not observed on prescription costs and antibiotics use. Analyses of the mechanism and long-term effect of public reporting of medicine use information are recommended for further studies.

Keywords: Quasi-experimental design, Propensity score, Township hospitals, Transparency, Rational drug use, China

\footnotetext{
*Correspondence: xpzhang602@163.com

School of Medicine and Health Management, Tongji Medical College,

HuaZhong University of Science and Technology, Wuhan, Hubei Province, China
} 


\section{Background}

Transparency has become one of the hottest topics in the health care system worldwide. The Institute of Medicine defined health care transparency as making health care available to the public in a reliable and understandable manner, with a primary focus on the reporting of information and processes [1]. In this study, we discuss public reporting of information. According to the literature, health care transparency can: (a) ensure that consumers make informed health care choices; (b) increase trust in the patient-physician relationship and health care systems; (c) improve performance (quality, safety, and efficiency) throughout the health care system because of competition and/or availability of clinical benchmarks [2,3]. Berwick proposed that public reporting can improve performance via two pathways, the selection and the change pathways [4]. In the selection pathway, patients or their intermediaries compare publicly released performance data and reward the better-performing providers by "selecting" (rewarding, recognizing, or paying) that provider. In the change (or quality improvement) pathway, performance data help providers identify areas in which they underperform and improve their performance accordingly [4-6].

In fact, public reporting of quality information is a growing movement in many countries. In US health care system, almost every state has quality reporting programs for hospitals, and the Center for Medicare and Medicaid Services has initiated public disclosure programs for hospitals, managed care plans, nursing homes, and home health agencies [7]. In South Korea, a national insurance review agency has been publicly releasing information about antibiotics use rates among health care organizations since 2006 [8]. Some other countries have also attempted to develop and collect quality measures that capture consumer experience with health care systems [9]. In 2009, the Chinese government established the document "Views on Deepening the Reform of Medical Care System", which points out the importance of increasing drug regulatory transparency. Certain links in the drug-use segment, such as antibiotics use, prescription comment, drug expenditure reimbursement, and so on, were very important parts of the regulation and were also the most urgent parts that have to be transparent. Many local governments had done certain attempts, but no results have been published yet.

As public reporting rapidly expands, several studies have examined the effects of information disclosure on quality in hospitals or nursing home markets. A review synthesized the evidence for using publicly reported performance data to improve quality, but found mixed results [5]. After that, other studies have been published. Won Mo Jang [10] found that repeated public releases on Caesarean section rates had no significant effect on reducing Caesarean section rates. In addition, Bundorf [11] evaluated a reporting program for Medicare HMOs and found that public reporting has no significant positive effect on quality. However, Rachel [12] found that public reporting in post-acute care setting had mixed effects in areas without public reporting, which improved in high-ranking facilities but worsened in low-ranking facilities. Jung also reported that release of quality information had a significant positive effect on the quality in HMO markets during the earlier years of the voluntary disclosure program. Hence, the effect, if any, these activities have on quality improvement is still unclear.

In view of methodology, few studies used quasiexperimental method or had control groups. In Bundorf's study, although a comparison group was set, a new policy was implemented during the study period. Jung's study also had a control group, but it involved secondary data analysis in which the confounding factor was not matched. Hibbard [13] used a quasi-experimental method and set control group, but the control group was not randomly selected. Hence, a high-quality experimental or quasi-experimental design is needed to determine whether public reporting of information has an effect on quality improvement.

In our study, a quasi-experimental design method was used to explore whether public reporting of medicine use information can improve the doctor's prescribing practice in City Y, Hubei Province of China. The results can enrich the evidence of health care transparency on quality and provide references for China and other countries to promote health care transparency and rational drug use.

\section{Methods}

\section{Study design and setting}

A quasi-experimental design was used, and the intervention was implemented in November 2013. Our study was conducted in City Y, a city located in the central part of Hubei Province, China. Twenty township hospitals (total 22) were selected as intervention group or control group. First, Qianjiang had a total of 22 township hospitals. On the basis of hospital characteristics, including service population, number of doctors and outpatients, and revenue training times on rational drug use, the 22 hospitals were numbered from 1 to 22 using technique for order preference by similarity to an ideal solution (TOPSIS) method, and the first 20 hospitals were chosen as the sample. Second, starting with the first hospital, the two hospitals that had the nearest comprehensive evaluation index were chosen as a pair. Hence, a total of 10 pairs were formed. Third, coin tossing method was used to divide the two hospitals in each pair into the intervention group and the control group. In the end, each group had 10 hospitals. 


\section{The intervention}

Medicine use information was made public to the patients and doctors in the intervention group, including the values and rankings of average expenditure per prescription, percentage of prescriptions requiring antibiotics, and percentage of prescriptions requiring injections of each hospital and each doctor who had the privilege of prescribing medications. First, the medicine use information was calculated using the data from the information system of the Health Bureau of City Y. To ensure the correctness of the calculations, six researchers participated in this work and checked one another. Second, the information was printed in the appropriate format in A3/A4 paper. Third, the researchers posted the A3 papers on the bulletin board in the hall of hospitals and distributed the A4 papers to each doctor and director of the hospitals monthly.

According to Berwick's theory, the intervention's effects might come from three ways: (1) patient's rewarding the better-prescribing doctors after seeing the medicine use information; (2) pressure from the director of each hospital to improve their ranking; (3) doctors' effort to improve prescription behaviors to gain more patients or better reputation. However, in this paper, we do not distinguish the specific way from which the effect comes.

\section{Data sources}

First, in the baseline, the characteristics of all the doctors working in the hospitals including gender, age, experience, education level, title, and monthly income were surveyed. Second, the doctors' prescribing information was obtained from the information system of the Health Bureau of City Y each month provided by professional information workers. Third, the doctors whose prescriptions were more than 30 before (July to October 2013) and after (November 2013 to February 2014) the intervention were selected to be included in the analysis. Finally, 274 doctors were analyzed in this study, and all the prescriptions of each doctor each month were calculated to reflect each doctor's prescription behavior.

To ensure quality during data collection, a survey guide was set, and all the researchers were trained according to the guide before the survey. A quality supervisor was also assigned to check the work every day. For the quality assurance of data collection of the doctors' prescription information, professional information workers were asked to export data according to the format set, and a special computer was used to store the data. All the data used in this study was not freely available and we got the permission of the Health Bureau of City Y.

\section{Statistical variables}

A matched-pair design with PSM was used to analyze the effects of the intervention on the doctors' prescribing behavior. Three indicators were used: prescription costs, antibiotics use, and injection use. All the three indicators were defined as two categorical variables. If the average expenditure for the prescription of a doctor after the intervention was lower than before, his or her prescription cost was designated as a "decrease", otherwise (if the expenditure remained the same or was higher), it was designated as a "not decrease". If the percentage of the prescriptions of a doctor requiring antibiotics after the intervention was lower than before, his or her antibiotics use was defined as a "decrease", otherwise, a "not decrease". If the percentage of the prescriptions of a doctor requiring injections after the intervention was lower than before, his or her injection use was defined as a "decrease", otherwise, a "not decrease".

\section{PSM}

To balance the covariate difference between the intervention group and the control group doctors, PSM was used to evaluate the effects of intervention by comparing the outcomes in the intervention group with the results in the control group. Control group doctors are suitable matches for intervention group doctors if they have similar observed characteristics as evaluated by a particular distance metric, i.e., the propensity score from a logistic regression model used in this study (Table 1 ).

PSM was implemented in two steps: (1) the conditional probability of being highly compensated (i.e., the propensity score) was calculated; and (2) the scores in close proximity to the exposed propensity scores were selected from the control group. Nearest-neighbor matching was performed using a caliper value of 0.03 [14], and caliper matching with one-to-one matches based on the propensity scores was used in this study.

\section{Table 1 Description of variables in the logistic model of} PSM

\begin{tabular}{|c|c|}
\hline Variable name & Description \\
\hline \multicolumn{2}{|l|}{ Dependent variable } \\
\hline Group & intervention group (1); control group (0) \\
\hline \multicolumn{2}{|l|}{ Independent variable } \\
\hline Gender & male (1); female (0) \\
\hline Age (year) & continuous \\
\hline Experience (year) & continuous \\
\hline Education level & $\begin{array}{l}\text { high school or less (1); some college or } \\
\text { associates (2); bachelors or higher (3) }\end{array}$ \\
\hline Title & $\begin{array}{l}\text { not certified (1); certified doctor (2); house } \\
\text { physician (3); doctor in charge of a case (4); } \\
\text { assistant director physician and above (5) }\end{array}$ \\
\hline Monthly income (yuan) & $\begin{array}{l}<1500(1) ; 1501-2000(2) ; 2001-2500(3) ; \\
2501-3000(4) ;>3001(5)\end{array}$ \\
\hline
\end{tabular}




\section{Data analysis}

Independent sample $t$-test or chi-square test was used to analyze the differences of the covariates between the intervention and control groups before and after PSM. $t$ test and chi-square test were used to estimate the association between the intervention and the result indicators, including prescription costs, antibiotics use, and injection use. Statistical significance was accepted at p-value $\leq 0.05$ (SPSS 12.0). The study was approved by the Committee of Tongji Medical College, HuaZhong University of Science and Technology (IORG No: IORG0003571), and the 274 doctors all provided written informed consent.

\section{Results}

\section{Characteristics of the participating doctors}

The average expenditure per prescription of the 274 doctors before the intervention was $42.82 \mathrm{RMB}$ yuan (USD 6.97, the exchange rate was US $\$ 1=$ RMB 6.14 in March 2014) and after the intervention was 46.68 RMB yuan (USD 7.60). The percentages of prescriptions requiring antibiotics of the 274 doctors before and after the intervention were found to be $63.00 \%$ and $61.36 \%$, respectively. The percentages of prescriptions requiring injections of the 274 doctors before and after the intervention were found to be $70.79 \%$ and $66.02 \%$, respectively. Among the 274 doctors, 68 doctors (24.82\%) reduced the average expenditure per prescription, 123 doctors $(44.89 \%)$ reduced the percentage of prescriptions requiring antibiotics, and 192 doctors (70.07\%) reduced the percentage of prescriptions requiring injections (Table 2).

\section{Propensity score estimation}

The doctors' propensity scores were estimated using a logistic regression model of the probability of receiving the intervention based on the following: gender, education level, title, income, age, and experience. A total of 108 pairs were obtained after PSM. The utilization of the total sample was $79 \%$ (216 out of 274). Table 3 shows the covariate in the pre- and post-matched samples for the intervention and control groups. A significant difference was found between the income of the groups $(\mathrm{p}<0.05)$. After PSM, the difference was insignificant $(\mathrm{p}>0.05)$. Most variables obtained a high $\mathrm{p}$-value after PSM.

\section{Effect of the intervention on the doctors' prescribing behavior}

Table 4 shows that the average expenditure per prescription, percentage of prescriptions requiring antibiotics, and percentage of prescriptions requiring injections in the baseline were all comparable. After the intervention, a significant difference $(\mathrm{p}<0.05)$ for the percentage of prescriptions requiring injections was found between the intervention and control groups, but this difference did not exist for the
Table 2 Other characteristics of the investigated doctors

\begin{tabular}{ll}
\hline Variable name & Median \pm SD/frequency (percent) \\
\hline Gender & $172(62.77 \%)$ \\
Male & $102(37.23 \%)$ \\
Female & \\
Education level & $84(30.66 \%)$ \\
High school or less & $139(50.73 \%)$ \\
Some college or associates & $51(18.61 \%)$ \\
Bachelors or higher & \\
Title & $12(4.38 \%)$ \\
Not certified & $57(20.80 \%)$ \\
Certified doctor & $60(21.90 \%)$ \\
House physician & $130(47.45 \%)$ \\
Doctor in charge of a case & $15(5.47 \%)$ \\
Assistant director physician & \\
and above & \\
Monthly income (yuan) & $63(22.99 \%)$ \\
$<1500$ & $101(36.86 \%)$ \\
1501-2000 & $62(22.63 \%)$ \\
$2001-2500$ & $130(47.45 \%)$ \\
$2501-3000$ & $15(5.47 \%)$ \\
$>3001$ & $40 \pm 9.91$ \\
Age (year) & $19.5 \pm 12.25$ \\
\hline Experience (year) &
\end{tabular}

Note: The total number of sampled institutions was 274. For continuous and categorical variables, median \pm standard deviation and frequency (percent) were used, respectively.

indicators of average expenditure per prescription and percentage of prescriptions requiring antibiotics.

Table 5 clearly shows the changes in the doctors' prescription behaviors. After the intervention, 34.26\% of the doctors in the intervention group reduced the average expenditure per prescription, whereas $47.22 \%$ of the doctors in the control group reduced the average expenditure per prescription. Hence, no significant difference was shown between the two groups $(p>0.05)$. The ratio of the doctors who decreased the percentage of prescriptions requiring antibiotics in the intervention group was $45.37 \%$, which is higher than that in the control group (41.67\%), but no significant difference existed ( $p>0.05$ ). Of the doctors in the intervention group, 39.81\% reduced the percentage of prescriptions requiring injections, whereas $26.85 \%$ of doctors in the control group reduced the percentage of prescriptions requiring injections. These results exhibited a significant difference $(\mathrm{p}<0.05)$.

The results from Tables 4 and 5 reveal that after the intervention, more doctors reduced injection use, leading to the lower percentage of prescriptions requiring injections in the intervention group than in the control group. 
Table 3 Variables for per- and post-matched samples for the intervention and control group

\begin{tabular}{|c|c|c|c|c|c|c|}
\hline \multirow[t]{2}{*}{ Variable name } & \multicolumn{3}{|c|}{ Pre-PSM approach } & \multicolumn{3}{|c|}{ Post-PSM approach } \\
\hline & Control group & Intervention group & $P$ value & Control group & Intervention group & $P$ value \\
\hline \multicolumn{7}{|l|}{ Gender } \\
\hline Male & $92(64.34 \%)$ & $80(61.07 \%)$ & 0.576 & $68(62.96 \%)$ & 70 (65.74\%) & 0.670 \\
\hline Female & $51(35.66 \%)$ & $51(38.93 \%)$ & & $40(37.04 \%)$ & 38 (34.26\%) & \\
\hline \multicolumn{7}{|l|}{ Education level } \\
\hline High school or less & $46(32.17 \%)$ & $38(29.01 \%)$ & 0.837 & $37(34.26 \%)$ & $34(31.48 \%)$ & 0.927 \\
\hline Some college or associates & 70 (48.95\%) & $69(52.67 \%)$ & 0.752 & $50(46.30 \%)$ & 54 (50.00\%) & 0.733 \\
\hline Bachelors or higher (Refer) & $27(18.88 \%)$ & $24(18.32 \%)$ & & $21(19.44 \%)$ & $20(18.52 \%)$ & \\
\hline \multicolumn{7}{|l|}{ Title } \\
\hline Not certified & $5(3.50 \%)$ & 7 (5.34\%) & 0.199 & $5(4.63 \%)$ & $6(5.56 \%)$ & 0.433 \\
\hline Certified doctor & $25(17.48 \%)$ & $32(24.43 \%)$ & 0.123 & $20(18.52 \%)$ & 25 (23.15\%) & 0.282 \\
\hline House physician & $25(17.48 \%)$ & $35(26.72 \%)$ & 0.090 & $19(17.59 \%)$ & 27 (25.00\%) & 0.202 \\
\hline Doctor in charge of a case & 78 (54.55\%) & $52(39.69 \%)$ & 0.618 & $56(51.85 \%)$ & 45 (41.67\%) & 0.677 \\
\hline Assistant director physician and above (Refer) & $10(6.99 \%)$ & $5(3.82 \%)$ & & $8(7.41 \%)$ & $5(4.63 \%)$ & \\
\hline \multicolumn{7}{|l|}{ Monthly income (RMB yuan) } \\
\hline$<1500$ & $30(20.98 \%)$ & $33(25.19 \%)$ & 0.107 & $26(24.07 \%)$ & $33(30.56 \%)$ & 0.636 \\
\hline $1501-2000$ & 55 (38.46\%) & $46(35.11 \%)$ & 0.030 & $39(36.11 \%)$ & $41(37.96 \%)$ & 0.444 \\
\hline $2001-2500$ & 37 (25.87\%) & 25 (19.08\%) & 0.015 & $27(25.00 \%)$ & $20(18.52 \%)$ & 0.215 \\
\hline $2501-3000$ & $16(11.19 \%)$ & $13(9.92 \%)$ & 0.054 & $12(11.11 \%)$ & $7(6.48 \%)$ & 0.163 \\
\hline >3001 (Refer) & $5(3.50 \%)$ & $14(10.69 \%)$ & & $4(3.70 \%)$ & $7(6.48 \%)$ & \\
\hline Age (year) & $41 \pm 9.92$ & $38 \pm 9.86$ & 0.055 & $40 \pm 10.74$ & $39 \pm 9.43$ & 0.921 \\
\hline Experience (year) & $20 \pm 13.36$ & $18 \pm 10.82$ & 0.088 & $20 \pm 11.62$ & $20 \pm 10.19$ & 0.822 \\
\hline
\end{tabular}

Note: For continuous and categorical variables, median \pm standard deviation and frequency (percent) were used, respectively. Differences between groups were tested by independent $t$ test (Mann-Whitney $\mathrm{U}$ test) for continuous variables and by chi-square test for categorical variables. All $p$-values were two tailed.

\section{Discussion}

To the best of our knowledge, this study is the first attempt to evaluate the effect of publicly reporting medicine use information on rational drug use in China. The results show that before the intervention, the average

Table 4 Effect of the intervention on doctors' prescribing behavior after intervention (Median \pm SD)

\begin{tabular}{|c|c|c|c|c|}
\hline Variable name & $\begin{array}{l}\text { Intervention } \\
\text { group } \\
(\mathrm{N}=108)\end{array}$ & $\begin{array}{l}\text { Control } \\
\text { group } \\
(N=108)\end{array}$ & $\mathrm{z}^{\mathrm{a}}$ & $\begin{array}{l}P \\
\text { value }\end{array}$ \\
\hline
\end{tabular}

Average expenditure per

prescription of a docto

(RMB yuan)

$50.07 \pm 24.90 \quad 43.62 \pm 34.41 \quad-2.530 \quad 0.110$

Percentage of

prescriptions requiring

antibiotics of a doctor (\%)

$62.49 \pm 21.64 \quad 60.97 \pm 25.86 \quad-0.107 \quad 0.915$

Percentage of

prescriptions requiring

injections of a doctor (\%)

$64.66 \pm 23.51 \quad 70.52 \pm 25.57 \quad-2.349 \quad 0.019$

Note: Non-parametric tests were used because all the variables were not normal; a stands for Mann-Whitney $\mathrm{U}$ test. expenditure per prescription of the 274 doctors was 42.82 RMB yuan (USD 6.97), the percentage of prescriptions requiring antibiotics of the 274 doctors was $63.00 \%$, and the percentage of prescriptions requiring injections of the 274 doctors was $70.79 \%$. According to a paper published in health policy and planning (2013) [15], by the end of 2011, the average expenditure per prescription in Hubei Province was 26.67 RMB yuan (USD 4.34) and the percentage of prescriptions requiring injections was 59\%. Hence, City Y has higher prescription expenditure and ratio of injection than the average in Hubei Province. However, the World Health Organization (WHO) recommended that the percentage of encounters with antibiotics should range from $20.0 \%$ to $26.8 \%$, and the normal range of percent of encounters with injections should be $13.4 \%$ to $24.1 \%$ [16,17]. Therefore, the drug use in City Y is irrational and needs urgent attention.

In our study, we demonstrated that public reporting of medicine use information has a significant effect on the injection use in the township hospitals of China. Public release of doctors' performance data can improve doctors' behavior in prescribing injections. From a policy perspective, our study showed significant implications 
Table 5 Effect of the intervention on result indicators

\begin{tabular}{|c|c|c|c|c|c|c|}
\hline Variable name & & Intervention group & Control group & Total & Chi-square value & $P$ value \\
\hline \multirow[t]{3}{*}{ Prescription costs } & Not decrease & $71(65.74 \%)$ & $57(52.78 \%)$ & $128(59.26 \%)$ & 3.759 & 0.053 \\
\hline & Decrease & $37(34.26 \%)$ & $51(47.22 \%)$ & $88(40.74 \%)$ & & \\
\hline & Total & 108 (100.00\%) & 108 (100.00\%) & 216 (100.00\%) & & \\
\hline \multirow[t]{3}{*}{ Antibiotics use } & Not decrease & 59 (54.63\%) & $63(58.33 \%)$ & $122(56.48 \%)$ & 0.301 & 0.583 \\
\hline & Decrease & 49 (45.37\%) & $45(41.67 \%)$ & $94(43.52 \%)$ & & \\
\hline & Total & 108 (100.00\%) & $108(100.00 \%)$ & $216(100.00 \%)$ & & \\
\hline \multirow[t]{3}{*}{ Injection use } & Not decrease & 65 (60.19\%) & 79 (73.15\%) & 144 (66.67\%) & 4.083 & 0.043 \\
\hline & Decrease & $43(39.81 \%)$ & $29(26.85 \%)$ & $72(33.33 \%)$ & & \\
\hline & Total & 108 (100.00\%) & $108(100.00 \%)$ & $216(100.00 \%)$ & & \\
\hline
\end{tabular}

for the policymakers who were continuously searching for evidence of regulatory measure. Making doctors' and hospitals' information on injection use open would improve the doctors' prescription behavior and promote rational drug use. However, the mechanism on how public reporting of medicine use information affected injection use is not included in this paper and thus requires further studies.

Although Berwick thought that the effect came from the selection of consumers and the self-effort of providers to improve [4], certain barriers may still exist. Certain researchers believe that the selection of consumer pathway may be interrupted by the low level of awareness about the information [18-20]. For example, a research found that only $12 \%$ of patients consider the information released [21]. In our study, best effort was exerted to disseminate the information in an appropriate format and manner that was understandable and would motivate the patients to act on the information; however, the patients' comprehension may be insufficient, which became a barrier to consumers' selection [22,23]. Other researchers believe that to provide the best chance for success, the release of information should follow certain criteria such as accuracy, relevance, timeliness, costeffectiveness, and accountability [24]. Therefore, in future studies, evaluating the format or way of information disclosure may be done. Although we tried to communicate and convey medicine use information more effectively to patients, patients might have little or no choice but to go to the nearest hospital for reasons of district and policy issues in China. Furthermore, the incentive to sustain the effort of rational drug use can also affect doctor behavior [10]. In our study, we reported three indicators to patients and doctors, but we only saw the effect on injection use. Certain researchers found that the effect of disclosure on quality depended on the type of services $[25,26]$, and in our research, the results indicated the effect of disclosure on medicine use might depend on different indicators, which still need further research. These indicators might be the reasons why we did not see the effects of public reporting of medicine use information on prescription costs and antibiotics use.

From a methodological perspective, we have done a quasi-experimental design to eliminate the difference of institutions and used PSM to avoid the risks of selection bias based on doctors' characteristics in analyzing the effect of public reporting of medicine use information on rational drug use. By using logistic regression, the propensity score was estimated with the intervention as the outcome and the doctors' characteristics as the predictive variables. A total of 108 pairs of doctors shared close propensity scores, and similar characteristics were included in our study. The uniformity in the measured risk factor distribution indicates that the distribution of unmeasured variables is balanced, although the propensity scores cannot remove hidden biases except to the extent that the unmeasured prognostic variables are correlated with the measured covariates used to compute the score. Therefore, efficient development of health care policies can be supported using propensity score analysis [27].

Our study also has certain limitations. First, the data analyzed in our study were obtained four months before the intervention and four months after the intervention; hence, long-term effect was not discussed. However, we believe that understanding the immediate effect of the intervention is also important and could provide useful implications. Second, we did not discuss the responses of both patients and doctors, which would better explain the findings in our research and needs further research. Third, we did not evaluate the format or way of information release. In addition, we did not include variables of the patients in our analysis. Even though some researchers used certain criteria, no indicator system has been reported, indicating that much work still needs to been done in this area.

\section{Conclusions}

Irrational use of drug is still a policy issue in township hospitals in our study area. This study is the first attempt 
to evaluate the effect of public reporting of medicine use information on rational drug use in China. After controlling the difference of hospitals' characteristics in designing and using PSM to partially adjust the selection bias of doctors' characteristics, we demonstrated that public reporting of medicine information could decrease the percentage of prescriptions requiring injections in township hospitals of China. However, this effect was not observed on prescription costs and antibiotics use. The findings enrich the evidence of health care transparency on quality and provide references for China and other countries to promote health care transparency. Analyses of the mechanism and long-term effect of public reporting of medicine use information are recommended for future studies.

\section{Competing interests}

The authors declare that they have no competing interests.

\section{Authors' contributions}

Xingping $Z$ made the concept and design of this study and revised the manuscript. Xiaopeng $Z$ conducted the studies, collected the data, analyzed the data, drafted and revised the manuscript. LW participated in the collection of data and revised the manuscript. All authors read and approved the final manuscript

\section{Acknowledgments}

This research is supported by the National Natural Science Foundation of China (71373092). The authors would like to thank the National Natural Science Foundation of China for funding this research, and the Health Bureau of City Y, as well as Yuqing Tang, Lianping Yang, Chunyan Yang, Xuan Wang, Xin Du, Shiru Yang, Xi Yin, Yuqi Xiong, Fangying Zhong, Chenxi Liu, Xiaofei Zheng, and Meng Wu for helping in our data collection.

Received: 29 May 2014 Accepted: 6 October 2014

Published online: 11 November 2014

\section{References}

1. Institute of Medicine: Crossing the Quality Chasm: A New Health System for the Twenty-First Century. Washington: National Academies Press; 2001.

2. Collins SR, Davis K: Transparency in Health Care: The Time Has Come. The Commonwealth Fund: 2006.

3. Colmers J: Public Reporting and Transparency. The Commonwealth Fund. January 2007.

4. Berwick DM, James B, Coye MJ: Connections between quality measurement and improvement. Med Care 2003, 41:130-138.

5. Fung CH, Lim YW, Mattke S, Damberg C, Shekelle PG: Systematic review: the evidence that publishing patient care performance data improves quality of care. Ann Intern Med 2008, 148(2):111-123.

6. Marshall MN, Shekelle PG, Davies HT, Smith PC: Public reporting on quality in the United States and the United Kingdom. Health Aff 2003, 22(3):134-148

7. Jung K: The impact of information disclosure on quality of care in $\mathrm{HMO}$ markets. Int J Qual Health Care 2010, 22(6):461-468.

8. Health Insurance Review \& Assessment Service: Comprehensive Quality Report of National Health Insurance 2010. Seoul: 2010 [http://www.hira.or.kr/ eng/news/02/1212222_25619.html].

9. Chaudhry M, Gauld R, Horsburgh S: Hospital quality-of-care performance measurement and reporting: what New Zealand can learn from the United States and United Kingdom. N Z Med J 2012, 125(1366):51-63.

10. Jang WM, Eun SJ, Lee CE, Kim Y: Effect of repeated public releases on cesarean section rates. J Prev Med Public Health 2011, 44(1):2-8.

11. Bundorf K, Choudhry K, Baker L: Health plan performance measurement: does it affect quality of care for medicare managed care enrollees? Inquiry 2008, 45:168-183.

12. Werner RM, Konetzka RT, Kruse GB: Impact of public reporting on unreported quality of care. Health Serv Res 2009, 44(2 Pt 1):379-398.
13. Hibbard JH, Stockard J, Tusler M: Does publicizing hospital performance stimulate quality improvement efforts? Health Aff 2003, 22(2):84-94.

14. Austin PC: Some methods of propensity score matching had superior performance to others: results of an empirical investigation and Monte Carlo simulations. Biomet J 2009, 51:171-184.

15. Yang L, Liu C, Ferrier JA, Zhou W, Zhang X: The impact of the National Essential Medicines policy on prescribing behaviours in primary care facilities in Hubei province of China. Health Policy Plan 2013, 28(7):750-760

16. Isah AO, Ross-Degnan D, Quick J, Laing R, Mabadeje AFB: The Development of Standard Values for the Who Drug Use Prescribing Indicators. WHO; 2008 [http://archives.who.int/icium/icium1997/posters/1a2_txt.html].

17. WHO: Using indicators to measure country pharmaceutical situations: fact book on WHO Level I and Level II monitoring indicators. Geneva: WHO; 2006 [http://www.who.int/entity/medicines/publications/ WHOTCM2006.2A.pdf].

18. Khang YH, Yun SC, Jo MW, Lee MS, Lee SI: Public release of Institutional Cesarean section rates in South Korea: which women were aware of the information? Health Policy 2008, 86(1):10-16.

19. Marshall MN, Shekelle PG, Leatherman S, Brook RH: The public release of performance data: what do we expect to gain? A review of the evidence. JAMA 2000, 283(14):1866-1874.

20. Schneider EC, Epstein AM: Influence of cardiac-surgery performance reports on referral practices and access to care. A survey of cardiovascular specialists. N Engl J Med 1996, 335(4):251-256.

21. Schneider EC, Epstein AM: Use of public performance reports: a survey of patients undergoing cardiac surgery. JAMA 1998, 279:1638-1642.

22. Hibbard JH, Harris-Kojetin L, Mullin P, Lubalin J, Garfinkel S: Increasing the impact of health plan report cards by addressing consumers' concerns. Health Aff 2000, 19(5):138-143.

23. Hibbard JH, Slovic P, Peters E, Finucane ML: Strategies for reporting health plan performance information to consumers: evidence from controlled studies. Health Serv Res 2002, 37(2):291-313.

24. Clough JD, Engler D, Snow R, Canuto PE: Lack of relationship between the Cleveland Health Quality Choice project and decreased inpatient mortality in Cleveland. Am J Med Qual 2002, 17(2):47-55.

25. Mukamel DB, Weimer DL, Spector WD, Ladd H, Zinn JS: Publication of quality report cards and trends in reported quality measures in nursing homes. Health Serv Res 2008, 43(4):1244-1262.

26. Werner RM, Konetzka RT, Stuart EA, Norton EC, Polsky D, Park J: Impact of public reporting on quality of postacute care. Health Serv Res 2009, 44(4):1169-1187.

27. Tang $Y$, Zhang $X$, Yang $C$, Yang L, Wang H, Zhang X: Application of propensity scores to estimate the association between government subsidy and injection use in primary health care institutions in China BMC Health Serv Res 2013, 13:183.

doi:10.1186/s12913-014-0492-6

Cite this article as: Zhang et al: Application of propensity scores to explore the effect of public reporting of medicine use information on rational drug use in China: a quasi-experimental design. BMC Health Services Research 2014 14:492.

\section{Submit your next manuscript to BioMed Central and take full advantage of:}

- Convenient online submission

- Thorough peer review

- No space constraints or color figure charges

- Immediate publication on acceptance

- Inclusion in PubMed, CAS, Scopus and Google Scholar

- Research which is freely available for redistribution 